\title{
Bowel elastography - a pilot study for developing an elastographic scoring system to evaluate disease activity in pediatric Crohn's disease.
}

\author{
Otilia Fufezan1, Carmen Asavoaie', Attila Tamas', Dorin Farcau², Daniela Serban² \\ ${ }^{1}$ Department of Radiology, ${ }^{2}$ Department of Gastroenterology, Children Emergency Hospital, Cluj-Napoca, Romania
}

\begin{abstract}
The diagnosis and monitoring of Crohn's disease (CD) represents a diagnosis challenge in which imaging plays an important role. Aim: In the present paper we aim to demonstrate the role of sonoelastography (SE), performed in addition to hydrosonography (HS), in the evaluation of CD in children and to propose a scoring system for the appreciation of disease activity. Material and method: All the patients included into the study were diagnosed with CD and had underwent HS and $\mathrm{SE}$ as part of the imaging evaluation. In selected cases magnetic resonance enterography (MRE) was also performed. SE aspects were classified into three types, each corresponding to a specific bowel wall pattern: normal or remission (type A), inflammation (type B) and fibrosis (type C); this classification represents the basis of the scoring system. For the purpose of statistical analysis each evaluated bowel segment became an individual case. Results: Forty eight bowel segments were evaluated by SE: 21 type A, 20 type B and 7 type C. Statistically significant correlations were found between the intestinal wall HS changes, presence of complications, activity markers and the SE score. The HS assessment of the periintestinal area correlated only partially with SE score, while certain SE scores also proved to be predictors for the presence of complications or for increased values of the disease activity markers. Conclusions: SE, along with HS, represents a reliable investigation in the correct diagnosis and monitoring of pediatric patients with CD and the SE scoring system may be introduced as a method for the assessment of disease activity.
\end{abstract}

Keywords: hydrosonography, sonoelastography, Crohn's disease, bowel, scoring system

\section{Introduction}

For any specific pathology, it is of the utmost importance to find a "perfect" imaging tool that will allow an accurate diagnosis and easy monitoring of the disease. In Crohn's disease (CD), any segment of the digestive tract can be affected, but the ileum, colon or both are most frequently involved [1]. According to the revised Porto Criteria by ESPGHAN (European Society of Pediatric Gastroenterology, Hepatology, and Nutrition), a single gold standard for the diagnosis of CD is not available. The accurate diagnosis should be based on a combination of history, physical and laboratory examination, esophagogas-

Received 02.09.2015 Accepted 06.10.2015

Med Ultrason

2015, Vol. 17, No 4, 422-430

Corresponding author: Otilia Fufezan, MD

Dept of Radiology, 3rd Pediatric Clinic,

2-4 Campeni Street,

400217, Cluj-Napoca, Romania;

Email: otilia.fufezan@gmail.com troduodenoscopy and ileocolonoscopy with histology, and imaging of the small bowel [2]. The most significant imaging findings include: irregular thickening of the intestinal wall, mainly the submucosa, mucosal ulcerations, loss of parietal stratification, or loss of haustration [3]. Periintestinal fat is also usually involved and there is enlargement of the mesenteric lymphnodes [3]. Several complications (fistula, abscess and stenosis) may also appear. [1]. CD can benefit from many imaging modalities: conventional Xray, ultrasonography (US), magnetic resonance enterography (MRE), positron emission tomography, endoscopic videocapsule, or advanced endoscopy evaluations $[1,2,4]$. In the past decade, in Romania, an increasing number of cases of $\mathrm{CD}$ have been diagnosed among the pediatric population. The correct diagnosis included endoscopy with histology, as well as laboratory and imaging studies. Imaging modalities in pediatrics are different from those among the adult population with the ALARA (As Low As Reasonably Achievable) principle being the main and most important objective to consider when imaging chil- 
dren [5]. US techniques have significantly improved in the last years allowing a highly accurate evaluation of the majority of the anatomical structures, including the digestive tract and its adjacent structures [6,7]. Sonoelastography (SE) is a new emerging technique and has been found to have more and more applications in current practice, including the evaluation of the bowel [4]. According to our knowledge this technique has not been yet applied to the current practice of pediatric $\mathrm{CD}$ management.

Since SE has been demonstrated to help differentiate inflammation from fibrosis in other pathologies, the idea of using it for the assessment of the bowel in children came naturally. It is possible and easy to apply it for children since their abdominal wall is thinner and the target structures are close to the transducer $[4,6]$.

The aim of this study is to determine whether SE of the bowel wall, in addition to hydrosonography (HS) of the colon is a useful tool in the assessment and monitoring of pediatric patients with $\mathrm{CD}$ and to propose a SE scoring system for the assessment of CD activity.

\section{Material and method}

\section{Patients}

The study was conducted prospectively, between October 2014 and July 2015, and included 14 hospitalized patients (10 males), ages between 6 and 17 years and 7 months (mean age \pm standard deviation: 12 years \pm 3 years and 8 months), diagnosed with $\mathrm{CD}$ at the Gastroenterology Department of the Emergency Children Hospital, Cluj-Napoca. All investigations, including the imaging assessment, were performed after the informed consent was obtained from the parents or the legal guardians of the patients and with theapproval of the local Ethics Committee.

The including criteria were represented by: age under 18 years old, a positive diagnosis of $\mathrm{CD}$ based on the clinical history, endoscopy, histology, and imaging investigations. Patients with different inflammatory bowel disease (IBD), other than $\mathrm{CD}$, uncertain $\mathrm{CD}$ diagnosis and patients in whom SE or HS assessment could not be performed in optimal parameters (noncompliance or contraindications for the enema, failure of SE evaluation due to artifacts produced by constant presence of fluid within the bowel, multiple vessels surrounding the affected segments, significant gas bowel content or a large quantity of intraperitoneal fluid) were excluded from the study.

\section{Imaging methods}

The equipment used for US evaluations was a Toshiba Xario V 2.0 ultrasound machine with a linear probe, frequencies ranging between 7 and $14 \mathrm{MHz}$. The MRE examinations were performed on a $1.5 \mathrm{~T}$ magnetic resonance equipment at Hiperdia Imaging, Cluj-Napoca.

\section{Colon HS}

Colon HS was used to determine the segments of the digestive tract affected by the pathological process (ileum, colon), to delineate colon and ileum wall, and to assess haustration and mucosal irregularities $[8,9]$. HS was performed after previous colon preparation (including 2 enemas a few hours before examination), with the colon filled with fluid and after emptying. The colon was filled by a rectal catheter $(8 \mathrm{Fr}$ ) and distended with body temperature water.

Bowel wall thickness $>3 \mathrm{~mm}$ was considered pathological and the stratification pattern was classified as follows: visualization of all the layers (stratification is present), blurred stratification with thickening of the submucosa and complete loss of stratification [6-8]. The presence of signal during color/power Doppler was considered a sign of disease activity. We evaluated vessels in the submucosa and the pattern of the vasa recta (fig 1). The appearance of the peri-intestinal area, peritoneal fluid collections, and CD's complications (stenosis, abscesses, fistulas) were also assessed during the examination. Reaction of the mesenteric fat and enlargement of the lymph-nodes surrounding affected intestinal segment were considered signs of inflammation $[3,6]$. The absence of luminal expansion with suprajacent dilatation of the bowel was interpreted as a sign of stenosis. Fistulas or abscesses were notified when linear hypoechoic trajectories, respectively delineated fluid collections, without Doppler signal inside were present in the thickened fat [3]. The complications were recorded as present or absent.

\section{SE of the intestinal wall}

SE was applied over the gray scale image after colon emptying in a color scale way. The examination was performed in a freehand compression style (by realizing

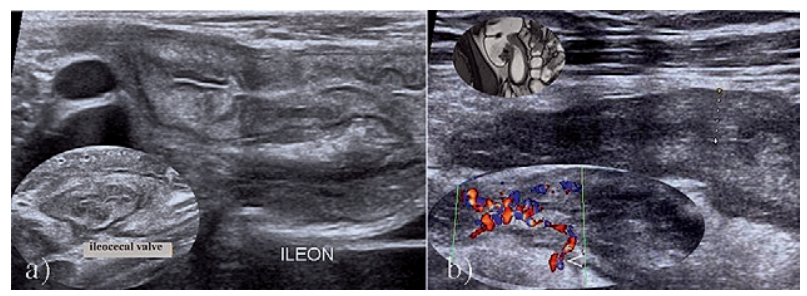

Fig 1. a) Gray scale US shows important inflammatory changes (bowel wall thickening with blurred stratification) at the level of the ileum (ILEON), ileocecal valve and cecum; b) Longitudinal US scan of the terminal ileum shows parietal thickening, areas with blurred or lost stratification. In the small oval icon from the bottom (color Doppler interrogation) there are vessels in the middle part of the wall (corresponding to submucosa) and vessels oriented perpendicular to the wall (vasa recta - arrowhead). The small icon from the top (T2 weighted MR evaluation) reveals thickening of the intestinal wall and ileocecal valve reaction. 
small and repetitive compression/decompression movements) with B-mode and SE images simultaneously displayed on a dual screen. The region of interest (ROI) was defined by the operator.

We conducted a qualitative evaluation of the intestinal wall, using the following color code: red - soft, green - intermediary stiffness, and blue - hard. SE findings were classified as follows (fig 2):

- Type A (normal bowel wall or wall in remission): in a normal or very mildly increased bowel wall thickness, with preserved stratification of bowel wall layers, SE revealed "colored stratification": mucosa and muscularis were blue and the submucosa was green (blue/green/blue pattern).

- Type $B$ (inflammatory wall): in a thick, regular or irregular wall, with preserved stratification, with or without hyperemia, the SE aspect was still "colored stratification", but with a different pattern: the submucosa was thicker and "harder" - green/blue; a thick wall with mildly blurred stratification, but

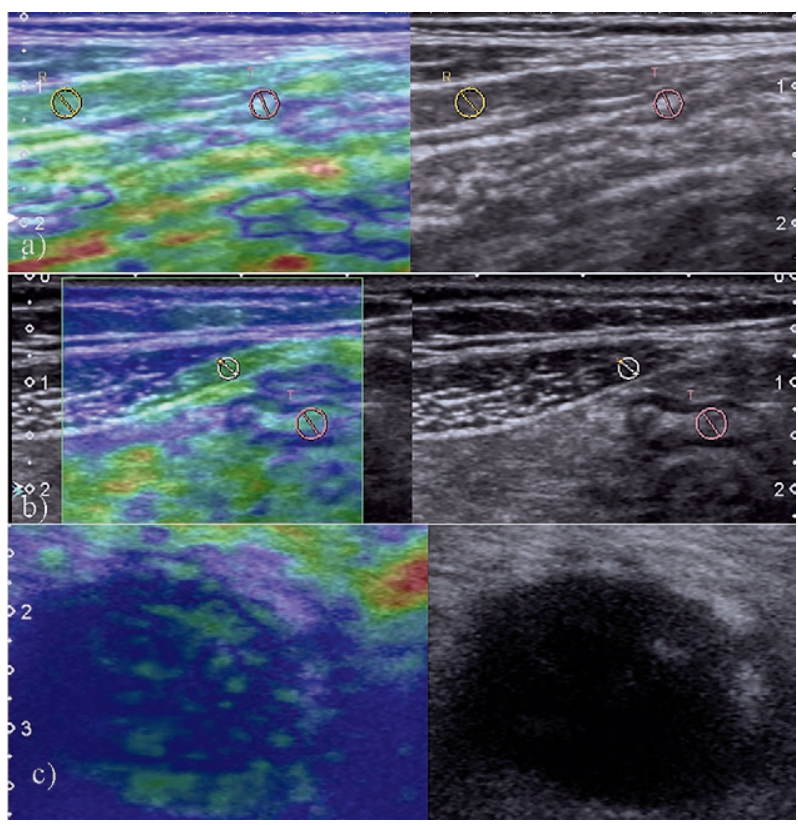

Fig 2. The 3 types of SE findings: a) normally stratified wall, with normal thickness in a longitudinal view of the colon, demonstrated a type A (blue/green/blue) "stratification" pattern; the strain of the colon and of the abdominal wall muscles were similar (equal ratio); b) type B intestinal wall: the "stratification" is preserved, but the submucosa is enlarged (mucosa and muscularis are blue and submucosa is green and thicker); strain measurements revealed higher values of the ileum wall as compared with the abdominal wall muscles. During visual analyses the surrounding tissues (mesenteric fat) appeared softer; c) Transverse colon with type C pattern (there is a "hard" appearance of the colon wall which is almost entirely blue); the surrounding tissue has a "soft" aspect. "green" aspect of the submucosa (supposition of inflammation) was still classified as type B.

- Type $C$ (fibrotic wall): if there was complete loss of stratification (none of the bowel wall layers could be identified) and SE revealed a completely or almost completely "blue" wall, the segment was classified as a type $\mathrm{C}$ and was considered consistent with fibrosis.

A semi-quantitative evaluation of the intestinal wall was also realized by performing strain ratio measurements. The target tissue was the affected intestinal wall at the level of submucosa or in the middle part of intestinal wall if stratification was lost. The muscles of the anterior abdominal wall represented the reference tissue for the strain measurements. The examination was initially performed on the longitudinal view, afterwards the transducer was rotated 90 degrees for the transverse view of the intestinal segment and the compressions were mild and regular. An equal ratio was considered normal or a sign of remission; a higher target strain as compared with the muscles represented inflammation and a lower target strain was interpreted as fibrosis (fig 3) [10,11].

As previously stated the SE examination was performed after colon emptying. The images were obtained with a flat intestinal lumen and we also tried to avoid large abdominal vessels from SE images. The last requirement was difficult for terminal ileum assessment because of the presence of iliac vessels and intestinal peristalsis. Whenever the intestinal lumen was distended with water or the iliac vessels were visible in the image the SE image was influenced and artifacts occurred. Therefore

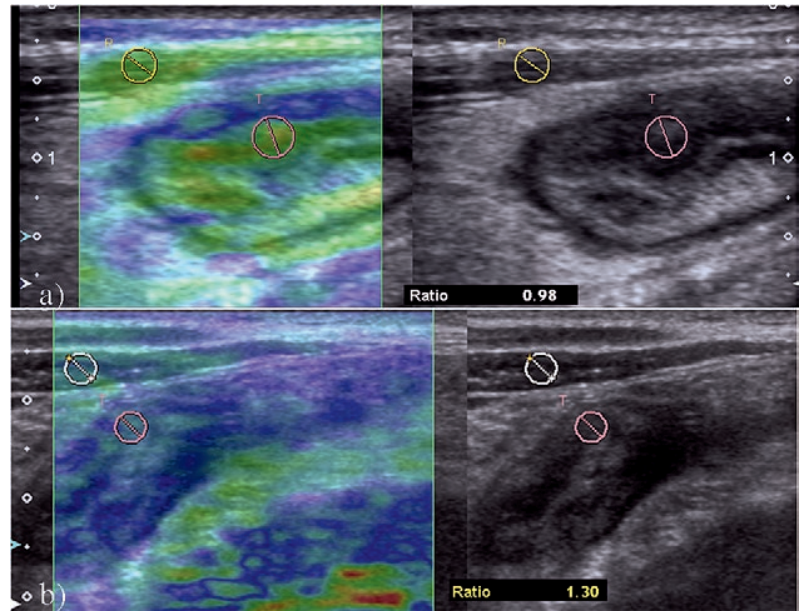

Fig 3. SE revealed a type B ileum segment with a strain similar with that of the abdominal wall muscles (a), with preserved stratification and thick submucosa and an ileum segment type $\mathrm{C}$ (b) with almost lost stratification (visual assessment reveals a "harder" appearance as compared with the surrounding fat and muscles; strain of the ileum was lower than that of the muscles). 
measurements obtained in such situations were excluded from the study. The artifacts are presented in figure 4 .

\section{Other studies}

In selected cases (6 patients) MRE was performed. MRE protocol consisted of bowel preparation by administration of Macrogol one hour before examination and i.v. contrast during the examination. Inflammation on MRE was documented by the presence of T2 hypersignal, ulcerations or ill-defined margins and contrast media uptake at the level of the mucosa. Fibrosis on the other hand was suggested by the pattern of contrast media enhancement and the presence of stenosis.

Colonoscopy and upper digestive tract endoscopy were performed in all patients.

$C$ reactive protein (CRP), erythrocyte sedimentation rate (ESR) and calprotectine (CAL) were also determined in all patients in order to assess disease activity. These were classified as increased or normal.

\section{Monitoring}

Some patients were diagnosed and treated before the present study started while others were diagnosed during this period. Also a selected number of patients received biological therapy while the study was going on. Therefore the bowel segments evaluated during the study actually represent various stages of the disease: at the diagnosis, after diagnosis and basic therapy, after biological therapy, in remission, during reactivations, and with complications. Since the main objective of the present

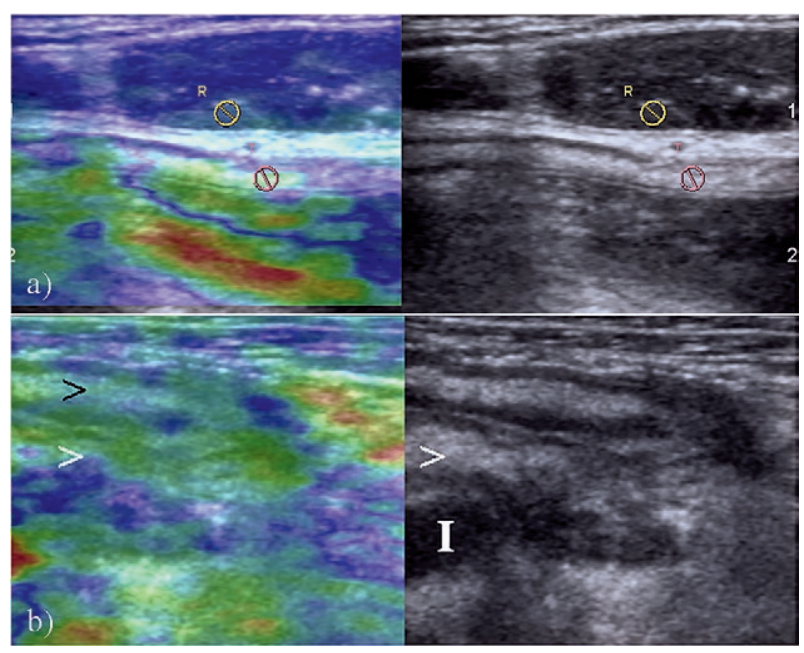

Fig 4. a) Longitudinal scan of the transverse colon during hydrosonography: SE applied on a segment filled with water (pink target ROI) was irrelevant because a correct compression could not be applied and therefore proper qualitative or strain evaluation was not possible. The abdominal wall muscle (yellow reference ROI) reveled a "hard" aspect during all examinations (the appearance was similar during compression and during relaxation). The RGB (red-green-blue) artifact is present in the luminal area. b) Assessment of ileum at the level of iliac vessels shows a type B SE pattern of the superficial bowel wall (black arrowhead): blue/green/blue aspect with prominence of submucosa, but the opposite wall of the ileum (white arrowhead) could not be evaluated by SE because of the artifacts produced by the presence of the iliac vessels (I); the appearance is blurred and the rim is undefined.

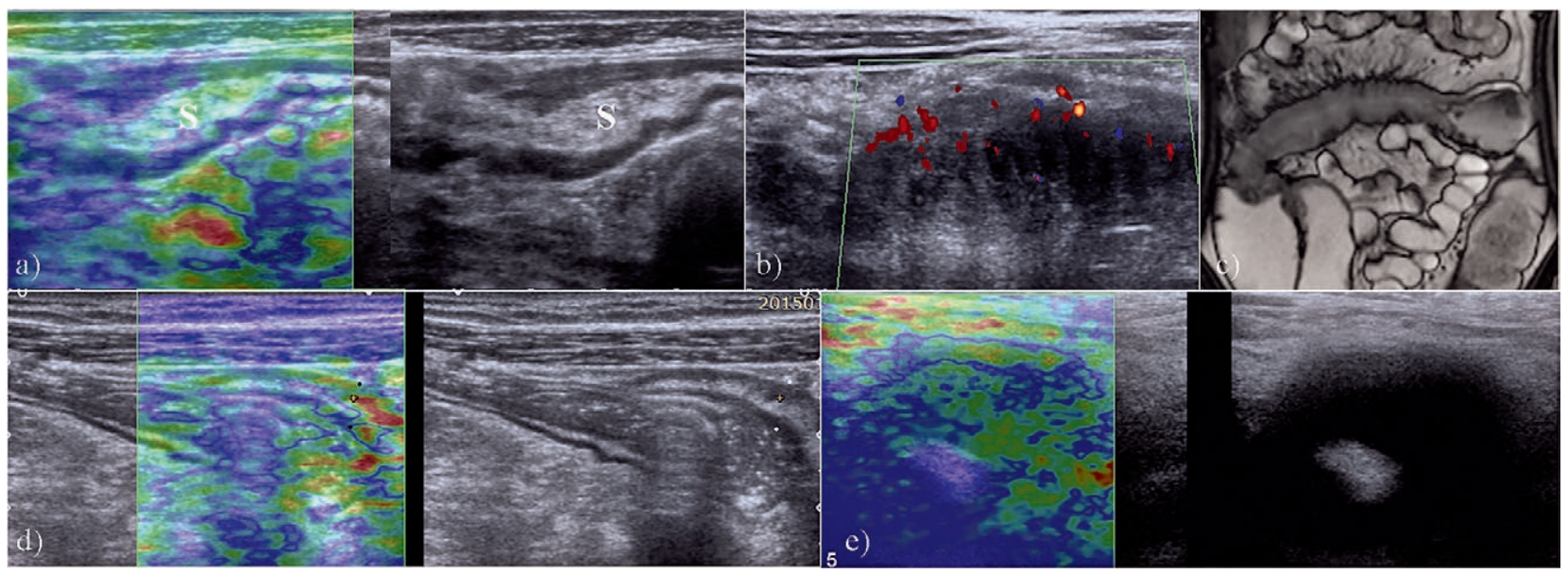

Fig 5. a) At diagnosis the descending colon showed important thickening with preserved stratification (type B pattern) and prominence of a "soft" submucosa (S); the strain measurements were not performed due to the presence of fluid; b) At the level of the transverse colon (longitudinal scan) stratification was completely lost and Doppler color interrogation reveals just a few vessels in the thickened wall; The gray scale findings and SE aspect performed at this level, presented in figure $2 \mathrm{c}$, are suggestive for stenosis with fibrosis. The MR study (c) confirmed these suspicions: T2 weighted coronal view revealed hyposignal of the bowel wall and an aspect of the transverse colon very similar with US findings; d) SE after 2 months of therapy revealed a spectacular improvement in the descending colon: parietal stratification and the SE aspect suggests a type A pattern; e) Less changes were detected on the transverse colon: on the gray scale examination the bowel wall appears very thick, with lost stratification and stenotic appearance; but the SE evaluation revealed an "improvement" regarding the elasticity of the colon (transverse view) - the visual evaluation was interpreted as having a softer aspect as compared with the previous assessment presented in figure $2 \mathrm{c}$. 
study was to demonstrate the role of HS and SE in the diagnosis and monitoring of patients with $\mathrm{CD}$, the case presented in figure 5 proves the role of these methods in both diagnosis and follow up.

\section{Statistical anlysis}

The statistical analysis was performed using MedCalc 12.5 software and the test that was applied was the ChiSquare test $(p<0.05)$. The US parameters considered for the statistical analysis were: bowel wall thickness, stratification, vascularity, periintestinal involvement (fat infiltration, enlarged lymph-nodes) and presence or absence of complications (stenosis, fistula, abscess). The biochemical parameters considered for the analysis were: CRP, ESR and CAL. The US parameters were combined and defined for the purpose of statistical analysis as criterion 1 (thickness + stratification), criterion 2 (thickness + vascularity) and criterion 3 (thickness + stratification + vascularity).

\section{Results}

We evaluated 48 bowel segments (30 ileum and 18 colon segments), from 14 patients with $\mathrm{CD}$, HS, and SE.
Six patients underwent MRE, while laboratory studies and endoscopy were performed in all patients.

In 3 cases $4 \mathrm{HS}$ and $\mathrm{SE}$ examinations were performed; in 3 patients 3 examinations were realized; 8 patients were evaluated twice, while one patient was assessed only once. The distribution of US characteristics and the biochemical data, in relationship with the SE scores and SR values, is presented in table I. The results are expressed in numbers of bowel segments.

Correlations between HS findings (thickening, stratification), color Doppler findings and a combination of these and the SE score (type A, B, C) and SR values were established (table II). The distribution of the US features in relation with SE and SR was statistically significant $(\mathrm{p}<0.005)$.

The relationship between the peri-intestinal alterations (fat infiltration, lymph nodes) and presence of complications with the SE and SR aspects was also studied (table III). The correlation between fat infiltration and SE or SR was statistically significant $(p<0.0001$ and $\mathrm{p}<0.0012$, respectively), but there was no statistically significant relationship between the presence of enlarged

Table I. Distribution of US criteria and biochemical data in relationship with the SE scores and SR values (results expressed in no. of bowel segments).

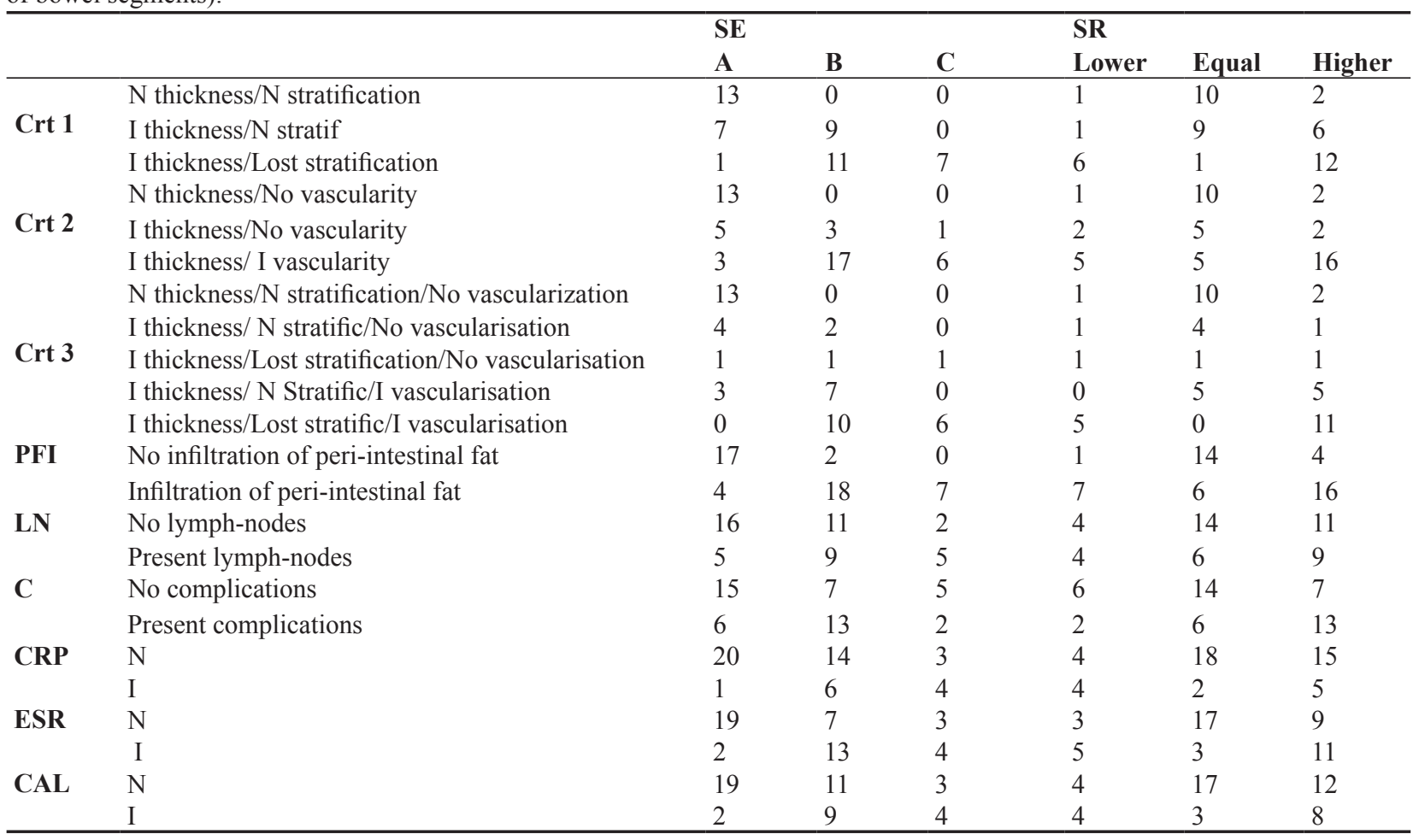

Criterion 1 - bowel thickness and stratification; Criterion 2 - bowel thickness and vascularity; Criterion 3 - bowel thickness, stratification, and vascularity; PFI - periintestinal fat infiltration; $\mathrm{LN}$ - lymph nodes; $\mathrm{C}$ - complications; CRP - C reactive protein; ESR - erythrocyte sedimentation rate; CAL - calprotectine; $\mathrm{SE}$ - sonoelastography score (A - normal, B - inflammation, $\mathrm{C}$ - fibrosis); $\mathrm{SR}$ - strain ratio; $\mathrm{N}$ normal; I - increased 
Table II. Correlations between US findings and SE and SR

\begin{tabular}{lllllll}
\hline & Crt 1 & Crt 1 & Crt 2 & Crt 2 & Crt 3 & Crt 3 \\
& SE & SR & SE & SR & SE & SR \\
\hline$\chi^{\mathbf{2}}$ & 33.95 & 19.27 & 28.18 & 13.76 & 37.50 & 22.58 \\
DF & 4 & 4 & 4 & 4 & 8 & 8 \\
p & $<0.0001$ & $<0.0007$ & $<0.0001$ & $=0.0081$ & $<0.0001$ & $=0.0039$ \\
\hline
\end{tabular}

Criterion 1 - bowel thickness and stratification; Criterion 2 - bowel thickness and vascularity; Criterion 3 - bowel thickness, stratification and vascularity; SE - sonoelastographic score; SR - strain ratio values; $\chi^{2}$ - chi square; $\mathrm{DF}$ - degrees of freedom

Table III. Correlations between the presence of periintestinal involvement and complications and SE and SR

\begin{tabular}{lllllll}
\hline & PFI & PFI & LN & LN & C & C \\
& SE & SR & SE & SR & SE & SR \\
\hline$\chi^{\mathbf{2}}$ & 26.93 & 13.39 & 5.39 & 1.37 & 6.29 & 6.34 \\
DF & 2 & 2 & 2 & 1 & 2 & 2 \\
$\mathbf{p}$ & $<0.0001$ & $=0.0012$ & $=0.0673$ & $=0.5025$ & $=0.0430$ & $=0.0418$ \\
\hline
\end{tabular}

PFI - periintestinal fat infiltration; LN - lymphnodes; $\mathrm{C}$ - complications; SE - sonoelastographic score; SR - strain ratio values; $\chi^{2}$ - chi square; DF - degrees of freedom

Table IV. Correlations between disease activity markers and SE and SR findings

\begin{tabular}{lllllll}
\hline & CRP & CRP & ESR & ESR & CAL & CAL \\
& SE & SR & SE & SR & SE & SR \\
\hline$\chi^{\mathbf{2}}$ & 9.12 & 5.26 & 14.23 & 8.79 & 8.55 & 4.48 \\
DF & 2 & 2 & 2 & 2 & 2 & 2 \\
$\mathbf{p}$ & $=0.0104$ & $=0.0721=0.0008=0.0123$ & $=0.0139$ & $=0.1065$ \\
\hline
\end{tabular}

CRP - "C" reactive protein; ESR - erythrocyte sedimentation rate; CAL - calprotectine; SE - sonoelastographic score; SR - strain ratio values; $\chi^{2}-$ chi square; $D F-$ degrees of freedom lymph-nodes and the SE and SR of the bowel $(\mathrm{p}=0.0673$ and $\mathrm{p}=0.5052$, respectively). The correlation between the presence of complications and the SE and SR was also found to be significant, but the correlation was not as strong ( $\mathrm{p}=0.0430$ and $\mathrm{p}=0.0418$ ).

A statistically significant correlation $(\mathrm{p}<0.05)$ was found between the disease activity markers (ESR, CRP and CAL) and the SE and SR findings, with the exception of CAL that did not significantly correlate with the SR values ( $p=0.1065)$ (table IV).

In a multivariate logistic regression analysis (stepwise) we identified that SE type B is an independent predictor for complications and SE and SR are predictors for increased levels of the disease activity markers. SE type $\mathrm{C}$ is a predictor for increased levels of CRP and SE type B and SR lower measurements represent combined predictors for elevated levels of ESR. An univariate logistic regression analysis additionally demonstrated that SE type B or SR type C represent predictors for elevated CAL (table V).

\section{Discussions}

SE has initially been used to assess breast lesions and liver fibrosis, but in the last decade this method was found to have many applications in a wide range of fields $[12,13]$. We propose the use of this US application to assess the ability of the intestinal wall to be compressed. There are a few articles in literature that reveal the possibility of evaluating the bowel by elastography, but to our knowledge the examinations have not been introduced in the current evaluation of pediatric $\mathrm{CD}$ patients and there

Tabel V. Sonoelastrography and strain ratio - predicting factors for complications and increased values of the disease activity markers.

\begin{tabular}{|c|c|c|c|c|c|}
\hline & & $\mathbf{S E}=\mathbf{B}-$ Complications & $\mathrm{SE}=\mathrm{C}-\mathrm{CRP}$ & $\mathrm{SE}=\mathrm{B} / \mathrm{SR}$ lower $-\mathbf{E S R}$ & $\mathrm{SE}=\mathrm{B} / \mathrm{C}-\mathrm{CAL}$ \\
\hline \multirow[t]{2}{*}{ Coeff } & & 1.53 & 1.86 & 2.82 & 2.05 \\
\hline & & & & 2.58 & 2.53 \\
\hline \multirow[t]{2}{*}{ Std dev } & & 0.62 & 0.86 & 0.87 & 0.86 \\
\hline & & & & 1.04 & 1.06 \\
\hline \multirow[t]{2}{*}{$\mathbf{p}$} & & 0.01 & 0.03 & 0.001 & 0.018 \\
\hline & & & & 0.013 & 0.017 \\
\hline \multirow{2}{*}{ Odds Ratio } & & 4.64 & 6.47 & 16.82 & 7.77 \\
\hline & & & & 13.28 & 12.66 \\
\hline \multirow[t]{2}{*}{$95 \%$ CI } & & $1.35-15.90$ & $1.17-35.58$ & 3.02 to 93.57 & 1.41 to 42.66 \\
\hline & & & & $1.70-103.6$ & 1.56 to 102.30 \\
\hline \multirow[t]{3}{*}{ ROC analysis } & AUC & 0.68 & 0.64 & 0.79 & 0.73 \\
\hline & SDT ERR & 0.07 & 0.10 & 0.06 & 0.07 \\
\hline & $95 \mathrm{CI}$ & 0.53 to 0.80 & 0.49 to 0.77 & $0.65-0.89$ & $0.59-0.85$ \\
\hline
\end{tabular}

$\mathrm{SE}=\mathrm{B}$ - sonoelastrographic type $\mathrm{B} ; \mathrm{SE}=\mathrm{C}$ - sonoelastrographic type $\mathrm{C}$; $\mathrm{SR}$ lower - strain ratio values lower than the those of the reference tissue (muscle of the anterior wall of the abdomen); CRP - C reactive protein; ESR - erythrocyte sedimentation rate; $\mathrm{CAL}-\mathrm{calprotectine;}$ CI - confidence interval.

SE type B - predictor for complications, SE type C - predictor for increased CRP, SE type B and SR lower - combined predictors for elevated ESR, SE type B or C - predictors for increased calprotectine 
have been no studies published yet demonstrating its role $[10,11]$.

In the management of $\mathrm{CD}$ the most important questions that need to be answered have to do with disease activity and differentiation between inflammation and fibrosis. This is usually done by determining the inflammation marker (CRP, ESR, CAL) and performing MRE examinations [14-17]. But in pediatrics MRE requires sedation and contrast media administration, which we try to minimize or avoid. Since US and especially HS is an important part of the evaluation of CD pediatric patients, we completed the examination with elastography to see if it could be used for the evaluation of CD activity and to differentiate between inflammation and fibrosis. Our study revealed a statistically significant correlation between the bowel wall thickness, stratification pattern, vascularisation, and SE score. This observation is important since both vascularisation patterns and SE give information on the activity of the disease.

Sjekavica et al found that the intensity of color Doppler and the resistance index in the arteries of the thickened bowel wall are important parameters in the evaluation of CD activity [18].

The present study shows that elastography brings additional information to those provided by color Doppler, because it proves that segments with increased vascularity may actually represent different stages of the pathological process: active inflammation (type $\mathrm{B}$ or higher $\mathrm{SR}$ ), progression towards fibrosis (type $\mathrm{C}$ or lower SR) or remission after treatment (type A or equal SR). A statistically significant correlation was also found between bowel wall thickening, the stratification pattern, color Doppler findings and the SE score and SR values. We believe that these findings suggest that the degrees of inflammation and fibrosis might be distinguished with elastography.

Inflammation and fibrosis can be quantified and graded using MRI. Rimola et al classified the different stages of inflammation and concluded that MRI accurately detects severe fibrosis based on the enhancement pattern [14]. In our study group there were 6 patients who underwent MRE, but the number of evaluated bowel segments was too small to statistically establish a correlation between elastography and MRE. Nevertheless since all of these patients had advanced forms of the disease the MRE findings proved to be accurately overlapping the SE and SR findings. In bowel segments, which were highly suggestive for fibrosis based on US findings, important thickening, loss of stratification, no vascularity, type $\mathrm{C}$ on SE, MRI diagnosed clear fibrosis. Therefore, we believe, based on these observations and some already published studies, that elastography may become in the future a re- liable tool in differentiating inflammation from fibrosis in $\mathrm{CD}$ patients. Future studies are required in order to accurately demonstrate these findings $[10,11]$.

Another important observation is that there were no bowel segments in our study group with normal thickness and lost stratification. That is because therapy is able to reverse the pathological process when the affected segment is inflamed or not entirely fibrotic, but not when there is true fibrosis

We also attempted to correlate the visual score and the SR values of the intestinal wall with the changes we found in the periintestinal area (fat infiltration and presence of lymph nodes close to the segment in question). There was a good correlation between the SE scores, SR values and the presence of fat infiltration. Almost all B type segments and all the $\mathrm{C}$ type segments were accompanied by infiltration of the periintestinal fat. This means that the worse the score was, the higher the probability of having fat infiltration. In the same manner most intestinal segments with SR values consistent with either fibrosis or inflammation were accompanied by fat infiltration and those segments that were in remission were surrounded by normal periintestinal fat. Neither the SE scores nor the SR values correlated with the presence of enlarged lymph nodes.

Complications represent an important issue in the diagnosis, therapy and monitoring of patients with $\mathrm{CD}$. It is known that in the weighted Pediatric Crohn's Disease Activity Index (wPCDAI), the presence of some complications is considered [19].

In children, most complications (stenosis, fistulas, abscesses) are visualized and accurately defined with HS and the additional use of color Doppler [6,9]. One of the most important limitations of HS has to do with the distinction between inflammatory stenosis and fibrotic stenosis. On grey scale they may appear similar, but we were able to notice in our study group that SE is able to distinguish between the two and our findings were confirmed by the MRE results [14].

Another important result regarding the relationship between SE and the presence of complications was that a type B on SE turned out to be a predictor of complications. Several inflammation markers were evaluated over the years for the diagnosis, differential diagnosis and for the assessment of CD activity. In our study we attempted to establish if there are correlations between the SE and SR results and some of these markers. In the era of biological treatment their importance is increased due to their potential to evaluate therapy response. Vermeire et al concluded that CRP is useful in the monitoring of CD as it correlates well with disease activity [20]. Additionally, Henriksen et al concluded that CRP values are pre- 
dictors for surgery in patients with CD [21]. ESR is also a reliable marker for the assessment of disease activity, but is less useful in clinical practice compared with CRP, due to its longer half-life and interference with other markers. Another important marker is represented by the faecal CAL. Burria et al showed that measuring faecal CAL can be highly useful in the diagnosis and management of IBD and that it could also predict disease course [22]. Additionally, a recent meta-analysis came to the conclusion that the addition of CRP and CAL to the clinical criteria improved the confident diagnosis of IBD [23].

We found that a type $\mathrm{C}$ on $\mathrm{SE}$ is a predictor for increased CRP values, that a type B on SE with a lower strain on SR represent combined predictors for high ESR and that both $\mathrm{B}$ and $\mathrm{C}$ types on SE are predictors for increased CAL. Since all of these are disease activity markers and are used for monitoring and treatment response assessment, our findings lead us to affirm that the SE scoring system may also be used for the evaluation of the disease activity and therapy response.

The main limitation of our study is represented by the small number of patients enrolled in the study and therefore small number of bowel wall segments that were evaluated. This of course meant an even smaller number of patients that underwent both elastography and MRE and prevented us from accurately evaluating the relationship between MRE findings and SE. We believe that establishing a significant correlation between MR and SE will help validate $\mathrm{SE}$ as reliable method for assessing disease activity. That needs to be the subject of future research. Since none of the patients from our study group underwent surgery during the time of the study, we were not able to establish any agreement between the histopathological findings and SE findings. In the era of biological therapy this will be a difficult task to accomplish since most patients will not end up having surgery. Other limitations of the study are related to the method itself. As expected only superficial bowel wall segments could be evaluated using SE, while the presence of intraluminal fluid and the presence of large vessels close to the affected bowel segment hindered the examination. Nevertheless, most of the time, these inconveniencies could be avoided. All US examinations were performed by only one examiner and therefore the intra/interobserver variability could not be evaluated.

In conclusion SE, performed in addition to HS, represents a reliable investigation in the correct diagnosis and monitoring of pediatric patients with $\mathrm{CD}$ and the SE scoring system may be introduced as a method for disease activity assessment. Further studies are needed to validate the technique as a modality to differentiate between inflammation and fibrosis.

\section{Conflict of interest: none}

Acknowledgements: The MRE examinations were interpreted by Dr. Cosmin Caraiani. We also want to thank Dr. Ionela Loredana Ciocan for her help in collecting and editing all the patient data.

\section{References}

1. Levine A, Griffiths A, Markowitz J, et al. Pediatric modification of the Montreal classification for inflammatory bowel disease: the Paris classification. Inflamm Bowel Dis 2011; 17: 1314-1321.

2. Levine A, Koletzko S, Turner D, et al; European Society of Pediatric Gastroenterology, Hepatology, and Nutrition. ESPGHAN revised porto criteria for the diagnosis of inflammatory bowel disease in children and adolescents. J Pediatr Gastroenterol Nutr 2014; 58: 795-806.

3. Casciani E, De Vincentiis C, Polettini E, et al. Imaging of the small bowel: Crohn's disease in paediatric patients. World J Radiol 2014; 6: 313-328

4. Anupindi SA, Podberesky DJ, Towbin AJ, et al. Pediatric inflammatory bowel disease: imaging issues with targeted solutions. Abdom Imaging 2015; 40: 975-992.

5. Civitelli F, Casciani E, Maccioni F, et al. Use of Imaging Techniques in Inflammatory Bowel Diseases That Minimize Radiation Exposure. Cur Gastroenterol Rep 2015; 7: 28.

6. Darge K, Anupindi S, Keener H, Rompel O. Ultrasound of the bowel in children: how we do it. Pediatr Radiol 2010; 40: 528-536.

7. Biko DM, Rosenbaum DG, Anupindi SA. Ultrasound features of pediatric Crohn disease: a guide for case interpretation. Pediatr Radiol 2015; 45: 1557-1566.

8. Maconi G, Radice E, Bareggi E, Porro GB. Hydrosonography of the gastrointestinal tract. AJR Am J Roentgenol 2009; 193: 700-708.

9. Nylund K, Ødegaard S, Hausken T, et al. Sonography of the small intestine. World J Gastroenterol 2009; 15: 13191330 .

10. Higgins PD, Dillman JR, Stidham RW, Johnson LA, Rubin JM. Ultrasound shear wave elastography detects bowel wall fibrosis in ex vivo human Crohn's disease specimens. J Crohns Colitis 20141 February. doi: http://dx.doi. org/10.1016/S1873-9946(14)60228-2

11. Havre RF, Leh S, Gilja OH, et al. Strain assessment in surgically resected inflammatory and neoplastic bowel lesions. Ultraschall Med 2014; 35: 149-158.

12. Garra BS. Elastography: current status, future prospects, and making it work for you. Ultrasound Q 2011; 27: 177-186.

13. Garra BS. Imaging and estimation of tissue elasticity by ultrasound. Ultrasound Q 2007; 23: 255-268.

14. Rimola J, Planell N, Rodríguez S, et al. Characterization of inflammation and fibrosis in Crohn's disease lesions by magnetic resonance imaging. Am J Gastroenterol 2015; 110: 432-440. 
15. Shenoy-Bhangle AS, Nimkin K, Aranson T, Gee MS. Value of diffusion-weighted imaging when added to magnetic resonance enterographic evaluation of Crohn disease in children. Pediatr Radiol 2015 Aug 4. doi: 10.1007/s00247015-3438-1.

16. Absah I, Bruining DH, Matsumoto JM, et al. MR enterography in pediatric inflammatory bowel disease: retrospective assessment of patient tolerance, image quality, and initial performance estimates. AJR Am J Roentgenol 2012; 199: W367-W375.

17. Mollard BJ, Smith EA, Dillman JR. Pediatric MR enterography: technique and approach to interpretation-how we do it. Radiology 2015; 274: 29-43.

18. Sjekavica I, Barbarić-Babić V, Krznarić Ž, Molnar M, Čuković-Čavka S, Štern-Padovan R. Assessment of Crohn's disease activity by doppler ultrasound of superior mesenteric artery and mural arteries in thickened bowel wall: cross-sectional study. Croat Med J 2007; 48: 822830 .
19. Turner D, Griffiths AM, Walters TD, et al. Mathematical weighting of the pediatric Crohn's disease activity index (PCDAI) and comparison with its other short versions. Inflamm Bowel Dis 2012; 18: 55-62.

20. Vermeire S, Van Assche G, Rutgeerts P. Laboratory markers in IBD: useful, magic, or unnecessary toys? Gut 2006; 55: 426-431.

21. Henriksen M, Jahnsen J, Lygren I, et al; IBSEN Study Group. C-reactive protein: a predictive factor and marker of inflammation in inflammatory bowel disease. Results from a prospective population-based study. Gut 2008; 57: 1518-1523.

22. Burri E, Beglinger C. Faecal calprotectin - a useful tool in the management of inflammatory bowel disease. Swiss Med Wkly 2012; 142: w13557.

23. Menees SB, Powell C, Kurlander J, Goel A, Chey WD. A meta-analysis of the utility of C-reactive protein, erythrocyte sedimentation rate, fecal calprotectin, and fecal lactoferrin to exclude inflammatory bowel disease in adults with IBS. Am J Gastroenterol 2015; 110: 444-454. 